\title{
Summer Edition 2019
}

\author{
Ronald G. Amedee, MD, FACS
}

Designated Institutional Official, Office of Graduate Medical Education, Division of Ochsner Academics, Ochsner Clinic Foundation, New Orleans, LA; Head of Clinical School and Professor, The University of Queensland Faculty of Medicine, Ochsner Clinical School, New Orleans, LA; Editor-in-Chief, Ochsner Journal

We might think we are nurturing our garden, but of course it's our garden that is really nurturing us.

$$
\text { -Jenny Uglow }
$$

This general medical topics edition of the Journal includes 4 original research manuscripts. Rice et al present "Building interventions to enhance pain care quality (BITE Pain) in medical surgical patients," a paper that will serve as a foundational work for future studies examining the use of nonopioid methods for postsurgical pain control. From the specialty of cardiology, another original research manuscript from Garcia et al- "Metaanalysis of multivessel vs culprit artery only percutaneous coronary intervention in ST elevation myocardial infarction"-presents the largest metaanalysis to date of randomized controlled trials studying multivessel $\mathrm{PCl}$ vs culprit artery only $\mathrm{PCl}$ in STEMI patients without shock. Moving a bit farther down the body, in "Superfluous amylase/lipase testing at a university-affiliated teaching hospital: a retrospective review," Hammami et al present the results of their examination of 8,801 lipase and amylase tests to determine if both tests-commonly ordered simultaneously-are necessary to diagnose acute pancreatitis.

In our Reviews and Contemporary Updates section are 4 manuscripts, including a paper by Thakolkaran and Shetty that offers valuable insights into the problem of "Acute hematogenous osteomyelitis in children," while Khemani et al offer their perspective on "Curative therapies for sickle cell disease." Kamli et al contributed an important paper that provides an overview of renal cell carcinoma, explains some reasons for therapy resistance in patients with RCC, and describes therapies that may overcome resistance to tyrosine kinase inhibitors. In "Congenital cytomegalovirus infection," Dietrich and Schieffelin shed light on a disease that causes more cases of permanent disability than better-known conditions such as Down syndrome and spina bifida and is the leading cause of nongenetic congenital hearing loss in high-income countries.

This issue also features 7 case reports, including papers by Robbins et al on "Peritoneal encapsulation with incarcerated Meckel diverticulum contributing to small bowel obstruction" (cover photo) and by Barton et al on "Delayed metastatic melanoma to the pharyngeal tonsil in an African American female."

Also featured in this issue are a Clinical Images case by Frischhertz et al entitled "Endovascular therapy for abdominal pregnancy" and a timely letter to the editor regarding how to use the perisurgical home to improve postoperative pain management and reduce opioid consumption. We also present an editorial by Mobley et al that details some of the frustration inherent in dealing with hypochondriacal patients but also presents some solid strategies for managing such situations.

Abstracts from the 10th Annual Evidence-Based Practice/Research Conference Evidence for Practice: Origins and New Directions Conference held on October 8, 2018 in New Orleans, LA, were published in the spring 2019 issue of the Ochsner Journal. Publication was made possible by sponsorship from Chamberlain University College of Nursing at Ochsner Health System. We are grateful to Chamberlain for their support of this important annual conference and for helping to ensure that the summaries of the research presented at the event are available online for worldwide dissemination.

When this edition publishes in mid-June, the summer growing season will be in full force throughout the United States. In the spirit of well-being, please take time to not only nourish your garden but also yourselves. Have a safe and happy summertime! 